\title{
Model-independent solution to dark energy problem
}

\section{Kuznetsov S.I.}

Web-Institute for Time Nature Explorations, Moscow, Russia;

E-mail: Kuznetsov<KSI@chronos.msu.ru>, <bitva@mail.ru>;

The proposed model-independent solution to the dark energy problem requires only redefining the redshift parameter $\mathrm{z}$ with no special assumptions of the physical mechanism of cosmological redshifting. The new definition of the redshift parameter finds its justification in cosmological models based on both special and general relativity. In this paper a Hubble diagram is produced from a sample of the SNe1a observational data, after recalculating measured redshifts according to the new definition with no change in corresponding magnitudes (apparent luminosities). A linear fit of the observational data obtained with the newly-defined redshift $z^{*}$ can be considered as an evidence for the nonaccelerating Universe with no need for any dark energy.

Keywords: Dark Energy, Redshift.

DOI: $10.18698 / 2309-7604-2015-1-276-288$

\section{Introduction}

As is known, the assumption of expansion of the Universe has been made on the basis of the observational fact that the spectral lines of light emitted by a distant galaxy are shifted to longer wavelengths. Quantitative characteristic of the observed increase in wavelength is the redshift parameter $z$. This dimensionless parameter is defined to be the fractional change in the wavelength of the emitted and detected light with respect to the one at emission. The equivalent definition of $z$ in terms of the photon frequency is not used in this article.

We consider two alternative approaches to explaining the nature of the cosmological redshift:

1. Standard cosmological model [1]. In this model based on the general relativity (GR) the growth of the wavelength of light from a cosmological source is a consequence of the expansion of space itself in the Universe.

2. Kinematic cosmology by Milne [2]. The origin of the observed cosmological redshift is the Doppler effect caused by the actual recession of galaxies in static space. The Kinematic cosmology is based on the special relativity (SR) that makes it alternative to the Standard cosmological model.

According to the empirical Hubble law [3] opened in 1929, the redshift is correlated with the distance to a galaxy considered as a cosmological light source. Graphical expression of the Hubble law is the diagram named after him. The linearity of the Hubble diagram means that the Universe is expanding uniformly, with a constant rate, so that the wavelengths of light from 
cosmological sources increase proportionally with their distances from Earth.

At the end of the last century redshift measurements were significantly advanced toward higher $z$. It became technically possible to observe supernovae of Type 1a (SNe1a) in distant galaxies. In 1998 it was discovered that the Hubble diagram on which the supernova magnitudes $M$ are plotted as a function of their redshifts $z$ (in logarithmic scale) deviates appreciably from the simple linear law for rather distant galaxies. The behavior of this deviation is that at large distances (for large $z$ ) galaxies look dimmer than expected at a constant rate of expansion (assuming no luminosity evolution in the look-back time). This discovery was the reason to put forward the hypothesis that the Universe was expanding slower in the past than is now. The cause of the acceleration of the Universe rate expansion has been referred to as dark energy. The origin of dark energy is unknown. And this is the problem.

There are many attempts to explain the SNe1a Hubble diagram and thus to solve the problem of dark energy [4]. Most of them involve an ad hoc hypothesis to explain the non-linearity of the Hubble diagram by the existence of a special mechanism of extra redshifting for electromagnetic radiation and(or) additional decrease in its luminosity (flux) at large cosmological distances.

The Standard cosmological model, for example, is forced to return the cosmological constant $\Lambda$ to the Einstein's equations, thus leading to anti-gravity effect between cosmological objects against the backdrop of gravitational attraction been weakening over time. However, this model gives rise to two serious problems known as the fine tuning and the cosmic coincidence [5].

As for the Kinematic cosmology an analytical expression fitting well the observational SNe1a data has been recently obtained in the framework of this theory even without invoking the concept of dark energy [6]. Unfortunately some additional assumptions made in this SR-model are unacceptable for models based on general relativity.

The purpose of this paper is to show that there is a solution to the problem of dark energy which does not depend on the intended physical mechanism of cosmological redshifting.

In our view, the desired solution of such a cosmological problem can be model-independent if it is constructed by using only measurable values without any free theoretical parameters and it admits physical interpretations on the ground of alternative theories as are general and special theories of relativity.

In the search for such a solution we have found that the conventional definition of redshift $z$ used in practice is incorrect. In the belief that it is the blunder which has led to the discovery of cosmic acceleration and to the hypothesis of dark energy we give a different definition of redshift 
parameter $\left(z^{*}\right)$ which we propose to call "squared redshift" or "red square". As the practically-used redshift $z\left(\equiv z^{p r}\right)$ the newly-defined parameter $z^{*}$ contains only measurable values but it does not distort the physical meaning of the theoretical determination of redshift $\left(\mathrm{z}^{\text {def }}\right.$ ) finding its justification in GR-models as well as in alternative SR-models. The Hubble diagrams $M(z)$ and $M\left(z^{*}\right)$ constructed for some sample of observational data on SNe1a are superimposed to comprise the rates of the Universe expansion in the old and new terms of redshift.

\section{Originally-defined and measurable redshift parameters}

The redshift parameter is a quantitative characteristic of wavelength growth of light that occurs in the expanding Universe between two events: its emission by a cosmological source and its registration by the observer on Earth.

In assuming that the detected photon with the measured wavelength $\lambda_{o b s}$ had the wavelength $\lambda_{e m}$ at the time of emission the redshift parameter is originally (theoretically) determined as follows:

$$
z^{d e f}=\frac{\lambda_{o b s}-\lambda_{e m}}{\lambda_{e m}}=\frac{\Delta \lambda}{\lambda_{e m}}
$$

or

$$
z^{d e f}+1=\frac{\lambda_{o b s}}{\lambda_{e m}}
$$

For calculating the redshift parameter it is necessary, according to definition (1), to measure the increase in the photon wavelength which occurs during its propagation from the instant of emission to that of registration and to divide the result by the initial (emitted) value of wavelength.

Unfortunately, the originally-defined redshift parameter $z^{\text {def }}$ is not measurable. Since the wavelength $\lambda_{e m}$ at the instant of emission $t_{00}$ (cosmic time from the beginning of the Big Bang) is unknown. Unlike $\lambda_{o b s}$, it can't be measured directly; only assumptions can be made with respect to its value. That is why in formula (1) the unknown wavelength $\lambda_{e m}$ which characterizes some observable spectral line as to be at the time of emission of the registered light (i.e. in the distant past epoch) is replaced by the standard value $\lambda_{0}$ measured in the laboratory on Earth at the present time $t_{0}$.

Thus, the parameter $z^{p r}$ to be measurable in practice is defined as follows: 


$$
z^{p r}+1=\frac{\lambda_{o b s}}{\lambda_{0}}
$$

The redshifts $z$ used in observational cosmology generally and those listed in the tables of observational data on Type 1a supernovae in particular are counted according to this definition (3). So, in this paper, by a redshift $z$ is meant one to be measurable $\left(z \equiv z^{p r}\right)$.

In what follows we show that the formula (3) distorts the physical meaning of the original definition (1) giving underestimated values for the redshifts to be correlated with the corresponding distances on the cosmological scale when constructing the Hubble diagram.

\section{A misconception about the measurable redshift parameter $\mathbf{z}\left(\equiv \mathbf{z}^{\text {pr }}\right)$ in GR-models}

As already noted, in GR-models (in particular, in the Standard cosmological model) the light wavelength growth is caused by the expansion of space and occurs gradually at the motion of photons from the source to the receiver. Of importance is to emphasize that in expanding space any standard measure of length (a ruler) varies over time synchronously with the lengths to be measured (wavelengths, in our case). So that the numerical values of these lengths expressed in scale units of a certain ruler remain unchanged.

Hence it follows that despite the expansion of space, the direct measurement of the wavelength of a spectral line produced near the Supernova at the time of emission $t_{00}$ would give a numerical value $N_{00}\left(\lambda_{00}\right)$ to be exactly equal to the numerical value $N_{0}\left(\lambda_{0}\right)$ of the standard reference wavelength of this line measured in the laboratory on Earth at the present time $t_{00}$.

So, we really have:

$$
N_{00}\left(\lambda_{00}\right)=N_{0}\left(\lambda_{0}\right)
$$

The Standard cosmological model argues that equality (4) allows to make the substitution $\lambda_{0} \rightarrow \lambda_{e m}$ in the original determination of redshift parameter $z^{\text {def }}$ (1) for obtaining the measurable values $z^{p r}$ according to expression (3). In our opinion, some misunderstanding lies here.

Let us express the redshift parameter $z\left(\equiv z^{p \eta}\right)$ as a result of measurements of the observed ( $\left.\lambda_{o b s}\right)$ and the standard $\left(\lambda_{0}\right)$ wavelengths (as is used in practice): 


$$
z^{p r}=\frac{N_{0}\left(\lambda_{o b s}\right)-N_{0}\left(\lambda_{0}\right)}{N_{0}\left(\lambda_{0}\right)} .
$$

This is the formula (3) in which both the measurable wavelengths to be compared ( $\lambda_{o b s}$ and $\left.\lambda_{0}\right)$ are expressed in terms of a length unit $[l]_{0}$ of the same ruler $N_{0}$ to be applied for measurements on Earth at the present time $t_{0}$.

As mentioned above the redshift parameter is to evaluate the growth of the observed wavelength $\left(\lambda_{o b s}\right)$ compared with the initial (emitted) wavelength $\left(\lambda_{e m}\right)$ rather than with the standard one $\left(\lambda_{0}\right)$.

We believe that the wavelength of the emitted photon was a standard wavelength $\lambda_{00}$ at $t_{00}$, i.e. $\lambda_{e m}=\lambda_{00}$. Really, we can consider $\lambda_{00}$ to be a standard laboratory wavelength (similar to $\lambda_{0}$ ) in the event that its measurement was provided at the moment $t_{00}$ near the Supernova with the ruler $N_{00}$. This is the meaning of the equality (4).

Using (4) we can obtain from (5) another expression for the parameter $z\left(\equiv z^{p r}\right)$ :

$$
z^{p r}=\frac{N_{0}\left(\lambda_{o b s}\right)-N_{00}\left(\lambda_{00}\right)}{N_{00}\left(\lambda_{00}\right)}
$$

In this formula, the registered wavelength $\lambda_{o b s}$ is compared with the emitted wavelength $\lambda_{e m}\left(\equiv \lambda_{00}\right)$ as required by the physically correct definition (1). However, the result of this comparison (numerical value of redshift) is at least metrologically incorrect since the quantities to be compared are measured by non-identical rulers. The fact is that, as we found out earlier, in GRmodels the ruler $N_{00}$ in the Supernova should be different from the Earth ruler $N_{0}$ because of the expansion of space. The unit of length $[l]_{00}$ of the ruler $N_{00}$ appears to be shorter than the corresponding length unit $[l]_{0}$ of the ruler $N_{0}$, i.e. $[l]_{00}<[l]_{0}$. This means that the numerator of expression (6) contains the difference between two values which are obtained by measuring with non-identical rulers and, for this reason, are expressed in the length units being equal in name only but different physically.

So, from the metrological point of view the number obtained with expression (6) is physically meaningless. Consequently, replacement $\lambda_{0} \rightarrow \lambda_{e m}$ leading to expression (5) which gives the same result (6) can not be considered as allowable.

If this mistake is ignored, the formula (3) used in practice will lead to underestimating the 
redshift parameters $z$ and, hence, the expansion rate of the Universe. So that the luminosity distance to a galaxy will be greater than that determined by its redshift. A reasonable conclusion will be that supernovae in distant galaxies look dimmer. And such illusion can be a basis for the dark energy hypothesis which has arisen in 1998 [7]. In short, we believe that the dark energy problem has been raised by the improperly defined redshift parameter $z$.

\section{Removing the misconception about redshift parameter in GR-models}

To assess properly the value of redshift we should measure the initial wavelength $\lambda_{e m}\left(\equiv \lambda_{00}\right)$ by the same ruler $N_{0}$ which is used for measuring the observed wavelength $\left(\lambda_{o b s}\right)$ and laboratory wavelength $\left(\lambda_{0}\right)$.

In our notation it looks like this:

$$
z^{*}=\frac{N_{0}\left(\lambda_{o b s}\right)-N_{0}\left(\lambda_{00}\right)}{N_{0}\left(\lambda_{00}\right)}
$$

or

$$
z^{*}+1=\frac{N_{0}\left(\lambda_{o b s}\right)}{N_{0}\left(\lambda_{00}\right)}
$$

We have designated this new redshift parameter by star because it differs from conventional $z\left(\equiv z^{p r}\right)$ by its numerical value and its physical meaning. Unfortunately, we are unable to carry out such measurements to be in the distant past and in the vicinity of the Supernova.

But this is not required. As can be seen from (7), (8) it is sufficient to express the initial wavelength $\lambda_{e m}\left(\equiv \lambda_{00}\right)$ in the current length units $[l]_{0}$ which as we assume are greater than the relevant units $[l]_{00}$ at the emission time $t_{00}$, namely:

$$
[I]_{0}=[I]_{00}\left(Z^{p r}+1\right) .
$$

The relation (4) is equivalent to the following expression:

$$
\frac{\lambda_{00}}{[I]_{00}}=\frac{\lambda_{0}}{[I]_{0}}
$$


Whence taking into account (9) and using only the current units of length $[l]_{0}$ we get

$$
\frac{\lambda_{00}\left(Z^{p r}+1\right)}{[I]_{0}}=\frac{\lambda_{0}}{[I]_{0}}
$$

Finally, in current units of length the desired value of the Supernova standard wavelength $\lambda_{00}$ of an emitted photon is expressed in terms of its Earth laboratory wavelength $\lambda_{0}$ as follows:

$$
\lambda_{00}=\lambda_{0} /\left(z^{p r}+1\right)
$$

Using (4), (8), (12) and taking into account that $z \equiv z^{p r}$, we find a link between the old $(z)$ and new $\left(z^{*}\right)$ redshift parameters:

$$
Z^{*}+1=(Z+1)^{2}
$$

For practical purposes the newly-defined parameter $z^{*}$ is desirable to be represented by the directly measured values. Such values are the observed $\left(\lambda_{o b s}\right)$ and standard laboratory $\left(\lambda_{0}\right)$ wavelengths, i.e. the same terms that are included in the conventional definition (3).

From expression (8), taking into account (12) and (3), we obtain

$$
z^{*}+1=\lambda_{o b s}^{2} / \lambda_{0}^{2}
$$

or

$$
z^{*}=\frac{\lambda_{o b s}^{2}-\lambda_{0}^{2}}{\lambda_{0}^{2}},
$$

So, the dimensionless parameter $z^{*}$ can be determined, for example, as follows: the relation of the difference of squares of the observed wavelength and the corresponding laboratory wavelength to the square of the laboratory wavelength. This newly-defined parameter $z^{*}$ can be called as "squared redshift" or, simply, "red square". 


\section{A misconception about the measurable redshift parameter $\mathbf{z}\left(\equiv \mathbf{Z}^{\mathrm{pr}}\right)$ in SR-models and its removing}

For SR-models (in particular, for Milne's Kinematic cosmology) with space being considered to be static the assumption that $\lambda_{0}$ (now) is equal to $\lambda_{e m}$ (then) seems to be natural since in such space the wavelength of the emitted light should not change as it propagates from the source to the detector. Unlike GR-models, in SR-models the ruler does not change its length but when measuring a standard wavelength $\left(\lambda_{0}\right.$ or $\left.\lambda_{00}\right)$ the relative velocity of the source and the detector must be kept in mind. By definition, a wavelength to be standard is obtained by measuring in the fixed reference frame, moreover both the source and detector should be at rest with respect to each other.

As in the case of GR-models two standard wavelengths corresponding to the observed wavelength $\lambda_{\text {obs }}$ should be distinguished:

1. Earth-standard wavelength $\lambda_{0}$ is measured in the laboratory with both the source and detector being at rest on Earth (reference frame $N V_{0}$ ).

2. Supernova-standard wavelength $\lambda_{00}$ refers to the case where both the source and detector of the light to be measured are assumed at rest relative to the Supernova (reference frame $N V_{00}$ ).

Physically, these two quantities being measured each in its own frame of reference are equal. In our notation, this equation looks like this:

$$
N V_{00}\left(\lambda_{e m}\right) \equiv N V_{00}\left(\lambda_{00}\right)=N V_{0}\left(\lambda_{0}\right)
$$

However, this is not to say that $\lambda_{00}=\lambda_{0}$ since these values are measured in different reference frames moving relative to each other with some non-zero velocity $V$. When measuring the supernova-standard wavelength $\lambda_{00}$ it must be borne in mind that the detector as well as the source should be fixed relative to the Supernova. Since the source on the Supernova recedes from Earth at the velocity $V$, and the detector on Earth must be stationary relative to the source, then in the reference frame associated with the Earth the detector should also move at the same velocity $V$ in the direction of SNe. With the detector moving towards the light emitted by the supernova we find that wavelength $\lambda_{00}$ thus measured is less than the Earth-standard wavelength $\lambda_{0}$ and is:

$$
\lambda_{00}=\lambda_{0} /\left(z^{p r}+1\right)
$$


which is equivalent to (12) obtained in the case of GR-models.

By substituting of $\lambda_{00}$ (17) for $\lambda_{e m}$ in (1), taking into account (3) and the identity $z \equiv z^{p r}$, we again arrive at (15). Whence, as in the case of GR-models, we obtain the same relationship (13) between the old $(z)$ and new $\left(z^{*}\right)$ redshift parameters which can be represented as:

$$
z^{*}=2 z+z^{2}
$$

\section{Fitting the SNe observational data}

Hubble's law essentially means equality of the luminosity distance obtained from the inverse square law and the distance defined by the corresponding redshift (the rate of expantion). In the absence of luminosity evolution and/or variations of the expansion rate of the Universe, Hubble diagram should be a straight line (at least in logarithmic coordinates). The Standard cosmological model recognizes a deviation from the straight line in the diagram "magnitude vs. redshift" obtained with SNe1a data as an indication of the accelerated expansion [7]. We believe that the cause of the discovered effect of the accelerating Universe is the metrologically incorrect definition (3) of redshift parameter $z\left(\equiv z^{p r}\right)$ which is used observational cosmology.

Having made adjustments to the definition of the measurable redshift parameter we can expect that the Hubble diagram $M\left(z^{*}\right)$ will be linear in new terms (15). According to the scale of luminosity distances adopted in observational cosmology such linear relationship can be analytically represented as follows:

$$
M\left(z^{*}\right)=M_{0}+5 \log _{10}\left(z^{*}\right)
$$

where $M_{0}$ is a constant.

In order to obtain the observational values of $z^{*}$ we can take available redshifts $z$ from the observational data and recalculate them by using the established relationship (18) between these two parameters. Due to the same relationship (18) the Hubble diagram in terms of old redshift $z$ ceases to be linear and should look like this:

$$
M(z)=M_{0}+5 \log _{10}\left(2 z+z^{2}\right)
$$

Precisely this analytical relationship was obtained in paper [6]. The derivation of this 
formula is based on the concepts of Milne's Kinematic cosmology and, notably, does not require the concept of dark energy. We will not comment on those additional assumptions that the author (F.Farley) has to do for reducing the apparent luminosity (magnitude) and obtaining the expression (20) which, as shown in the article [6], fits well the SNe1a observational data. It is important for us that this work lets have the numerical value of the constant $M_{0}$ at which this analytical formula (20) gives the best approximation of the observational data. This value is:

$$
M_{0}=41.8
$$

We believe that the constant $M_{0}$ calculated for (20) should have the same value (21) for our new representation of Hubble diagram (19) because only redshifts are changed in this relation with remaining magnitudes unaltered. Thus, due to the results of [6], we have the opportunity to build the Hubble diagram "magnitude $M$ vs. squared redshift $z$ " even without conducting statistical analysis of observational data to determine the constant $M_{0}$ which, incidentally, is the only free parameter in expressions (19) and (20).

To build the Hubble diagram in new values $z^{*}$ a sample of SNe1a observational data is produced from the papers [8], [9] (these data were used in [6]). A small portion of available data (113 points) was taken only to demonstrate that simply transforming the observed redshifts $z$ into the newly-defined values $z^{*}$ we can get the data points to be lying on a straight line.

Fig. 1 shows two SNe1a Hubble diagrams "magnitude - redshift": $M(z)$ (crosses) and $M\left(z^{*}\right)$ (diamonds). Both are constructed on the basis of the same sample (113 points) from the SNe1a observational data.

Upper solid line $M(z)$ is given by formula (20). It approximates the observational data in which the original values of redshift $(z)$ are stored (as in the paper [6]). Dashed straight line is optional and serves to demonstrate a deviation of observational data (with conventional redshifts z) from the linear Hubble law. It should be mentioned that similar deviations were recognized in 1998 and served as a basis for the hypothesis of the Universe expansion rate acceleration (assuming the existence of dark energy). 


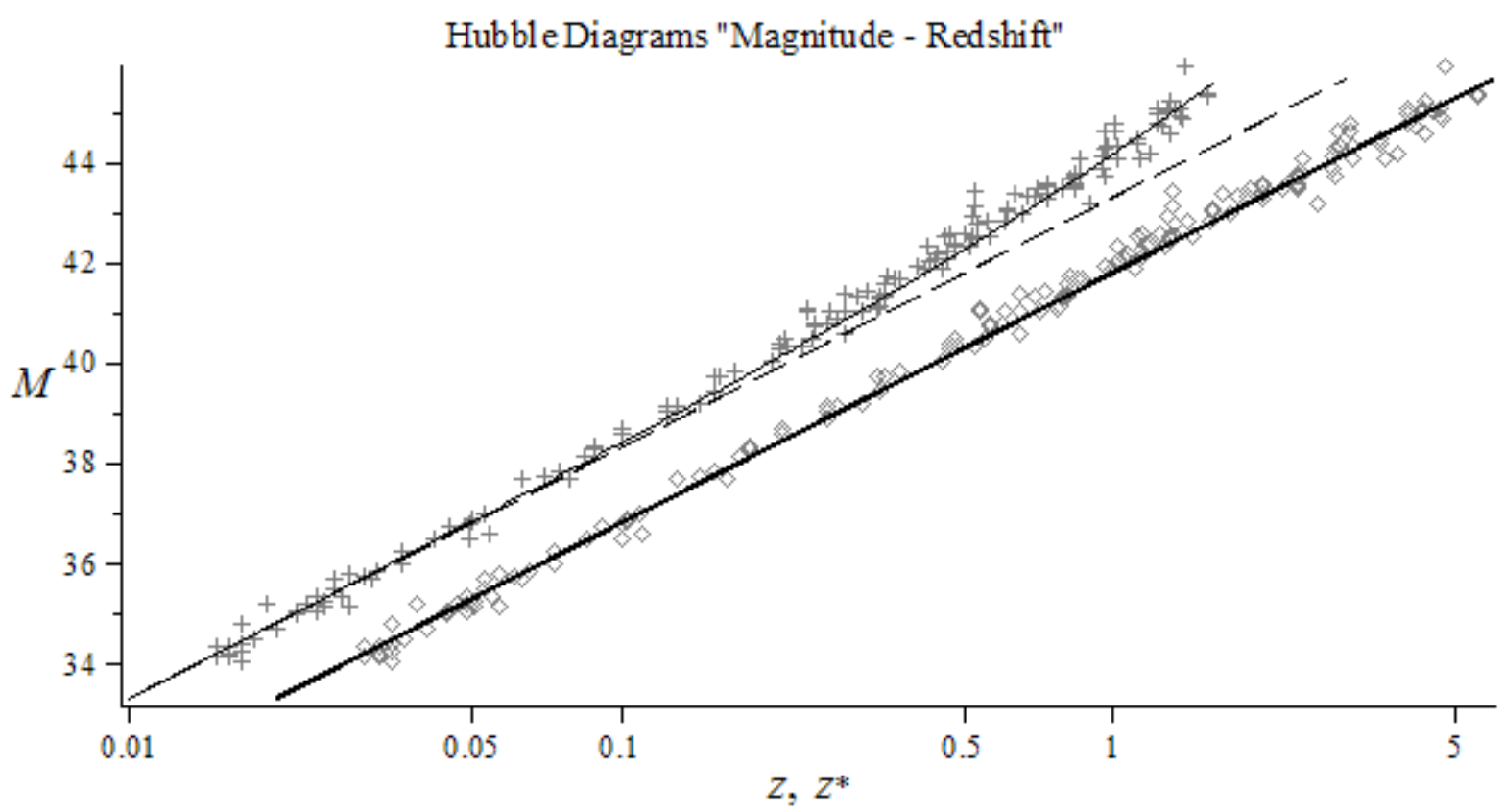

Fig.1. Two theoretical Hubble diagrams "magnitude - redshift" compared with observational data on SNela: 1) M(z) (upper solid line, crosses) and 2) M(z*) (lower solid line, diamonds). The redshifts $\mathrm{z}$ and $\mathrm{z}^{*}$ are in logarithmic coordinates.

Lower solid straight line defined by formula (19) is the expression of the Hubble law under the new definition of redshift parameter ("red square"). The observational data containing the recalculated values of redshift $\left(z^{*}\right)$ are shown as diamonds.

It should be recognized that in this paper we does not aim to assess how well the resulting formula (19) approximates observational data. This, we believe, is a special task that requires a serious statistical analysis and use of the entire body of observational data on supernovae. In this regard, we only refer to the second paper [10] by F.Farley in which a numerical estimation of the quality of his approximation (20) is given (in a somewhat different representation: instead of the argument $2 z+\mathrm{z}^{2}$ the expression $z+z^{2} / 2$ is used).

We emphasize that the graph $M\left(z^{*}\right)$ built on the SNe observational data is a straight line. As mentioned above the Hubble diagram "magnitude-redshift" being a straight line should be regarded as evidence of the lack of acceleration in the Universe. Such a diagram does not require any type of dark energy hypothesis for its explanation.

\section{Conclusion}

The problem of dark energy has been solved by a simple redefinition of the measurable 
redshift parameter $z$.

The newly-defined parameter $z^{*}$, which has been named "squared redshift" or "redsquare", has been involved in order to eliminate the mistake recognized in the conventional definition of $z$. This misconception is associated with incorrect assessment of the initial wavelength of the photon emitted from a cosmological source as it appears to the observer in the expanding Universe.

The proposed solution is model-independent since:

1) The red-square $z^{*}$ is a combination of two experimental (measurable) quantities, so there is no need to attract a specific physical mechanism of redshifting (i.e. a specific theoretical model).

2) The definition of red-square parameter is substantiated in alternative models such as the Standard cosmological model and the Milne's Kinematic cosmology. The Hubble diagram "magnitude $M$ - squared redshift $z^{*}$ is the same for these two models.

Namely, the linearity of Hubble diagram in terms of red-squares $z^{*}$ gives reason to rule out the hypothesis of the accelerating Universe with a mysterious dark energy.

If the proposed solution of the dark energy problem is considered to be correct only for an empty space universe then the situation in cosmology before 1998 will be restored when the current question was: is the Universe expansion slowing down under the influence of gravity? Taking this solution with its linear Hubble's law to be absolutely correct, physicists will be given the opportunity to concentrate on more difficult questions in cosmology. For instance: what is the gravitation?

\section{References}

1. Maroto A.L., Ramirez J. (2004). A Conceptual Tour About the Standard Cosmological Model. arXiv, astro-ph/0409280.

2. Milne E.A. (1948). Kinematic Relativity. Oxford.

3. Hubble E.P. (1929). A relation between distance and radial velocity among extra-galactic nebulae. Proc. Natl. Acad. Sci, Vol. 15, 168-173.

4. Copeland E.J., Sami M., Tsujikawa S. (2006). Dynamics of dark energy. Int. J. Mod. Phys. D., Vol. 15, 1753.

5. Carroll S.M. (2001). The Cosmological Constant. Living Reviews in Relativity. Vol. 4 (1).

6. Farley F.J.M. (2009). Alternative cosmology fits supernovae redshifts with no dark energy. arXiv, astro- ph/09013854.

7. Perlmutter S., Aldering G., Goldhaber G., Knop R.A., Nugent P., Castro P.G., Deustua S., 
Fabbro S., Goobar A., Groom D.E., Hook I. M., Kim A.G., Kim M.Y., Lee J.C., Nunes N.J., Pain R., Pennypacker C.R., Quimby R., Lidman C., Ellis R.S., Irwin M., McMahon R.G., RuizLapuente P., Walton N., Schaefer B., Boyle B.J., Filippenko A.V., Matheson T., Fruchter A.S., Panagia N., Newberg H.J.M., Couch W.J. (The Supernova Cosmology Project). (1999). Measurements of Omega and Lambda from 42 high redshift supernovae. Astrophysical J., Vol. $517(2), 565$.

8. Hicken M., Wood-Vasey W.M., Challis P., Jha S., Patrick L., Rest A., Kirshner R.P. (2012). Improved Dark Energy Constraints from 100 New CfA Supernova Type Ia Light Curves. arXiv, astro-ph/0901.4804.

9. Daly R.A., Mory M.P., O'Dea C.P., Kharb P., Baumont S., Guerra E.J., Djorgovski, S.G. (2009). Cosmological Studies with Radio Galaxies and Supernovae. Astrophysical Journal, 691 (2), 1058-1067.

10. Farley F.J.M. (2010). Does gravity operate between galaxies? Observational evidence reexamined. arXiv, astro-ph/10055052. 